\title{
RF ACCELERATING SYSTEM FOR A COMPACT ION SYNCHROTRON
}

\author{
K. Saito, K. Matsuda, H. Nishiuchi, M. Umezawa, K. Hiramoto, R. Shinagawa \\ Hitachi, Ltd. Power and Industrial Systems Group \\ 7-2-1 Omika-cho, Hitachi-shi Ibaraki-ken, 319-1221 Japan
}

\begin{abstract}
We have developed an RF accelerating system for a compact ion synchrotron. The RF cavity is an untuned type loaded with FINEMET cores, which have high complex permeability, high saturation flux density, and high Curie temperature properties. A new power feeding method, "multi-feed coupling," was developed to match the cavity impedance with output impedance of a solidstate RF amplifier. We have installed the RF accelerating system for a multi-purpose synchrotron at the Wakasawan Energy Research Centre in Japan. Ion beams were successfully accelerated by adjusting the RF control system using a direct digital synthesiser. For proton beam acceleration, dual-harmonics operation was effective to enhance the beam intensity due to reduction of the spacecharge effect.
\end{abstract}

\section{INTRODUCTION}

Recently, particle accelerators are being utilised for various applications in medical and industrial fields, not only for nuclear and high-energy physics. Such accelerators at hospitals or factory-based small facilities often have to be managed by a few operators who are non-experts regarding accelerators. Therefore, simple operation and easy maintenance are required for the accelerators along with compactness and low cost. We have designed and constructed a multi-purpose ion accelerator system for the Wakasa-wan Energy Research Centre in Japan, which consists of a compact synchrotron and a tandem-type electrostatic accelerator. One of the purposes of the accelerator system is for medical use in treatment of tumours, reflecting the situation that more than one fourth of all deaths in Japan are due to various kinds of cancers.

In order to make the accelerator system compact and inexpensive, injection energy of the synchrotron should be as low as possible, e.g. below $10 \mathrm{MeV}$ in the case of a proton beam. The beam dynamics is, however, strongly affected by the space-charge effect, and the resulting tune shift leads to emittance growth and beam loss through some betatron resonance. One method to mitigate the space-charge effect is "dual-harmonics acceleration" with a wide-band RF cavity producing a high voltage.

One of the complexities in the synchrotron operation comes from control of the RF cavity. We selected an untuned RF cavity, since its resonant frequency does not need to be tuned to the frequency of the supplied RF power. The control system of the RF cavity is therefore simple and an expensive power supply for a bias current to adjust permeability of loaded magnetic cores is unnecessary. Since complicated bias windings around the magnetic cores are also dispensable, the RF cavity becomes simple and compact.

In this paper, results of performance tests are described for the fabricated untuned RF cavity, together with those of the RF control system using a direct digital synthesiser (DDS). Results of beam acceleration tests are also presented, in which dual-harmonics acceleration was effectively performed.

\section{RF CAVITY}

The untuned RF cavity requires a Q-value low enough to cover the wide revolution frequency range of $0.5-8.5$ $\mathrm{MHz}$ and a gap voltage higher than $1 \mathrm{kV}$, in order to accelerate proton and heavy ion $\left(\mathrm{He}^{2+}, \mathrm{C}^{6+}\right)$ beams up to $200 \mathrm{MeV}$ and $55 \mathrm{MeV} / \mathrm{u}$, respectively. The proton beam is accelerated by dual-harmonics operation to enhance the beam intensity enough for treatment of cancer, e.g. higher than $10 \mathrm{nC} /$ pulse. The upper frequency limit of $8.5 \mathrm{MHz}$ of the superposed second harmonic is high enough to moderate the space-charge effect.

We selected FINEMET [1-2] as the material employed for the magnetic cores to realise the wide operation frequency range and the high accelerating gap voltage. FINEMET is a Fe-based soft magnetic alloy with an ultrafine grain structure, developed by Hitachi Metals, Ltd. It has high complex permeability of $(800,1500)$ around 3 $\mathrm{MHz}$, high Curie temperature of $570{ }^{\circ} \mathrm{C}$ and high saturation flux density of $1.35 \mathrm{~T}$. Excellent stability against excitation and heat-up by a high RF power has been confirmed with a testing cavity.

A photograph of the fabricated untuned RF cavity is presented in Fig. 1. The RF cavity has a single accelerating gap structure that consists of two quarter-

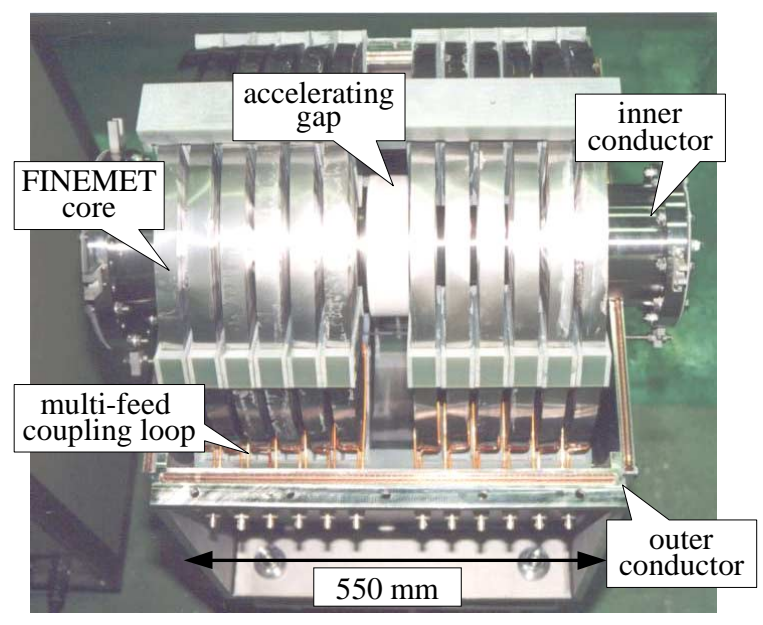

Figure 1: Photo of fabricated RF cavity 
wavelength coaxial resonators loaded with 12 FINEMET cores. The length of the RF cavity is only $550 \mathrm{~mm}$. The cavity impedance is designed as $700 \pm 150 \Omega$ in the operation frequency range of $0.5-8.5 \mathrm{MHz}$. For easy maintenance, core cooling is attained by air cooling with two blowers, and a solid-state amplifier is employed as an $\mathrm{RF}$ power source. Impedance matching between the cavity, feeding line and the power source is indispensable to produce a high voltage at the accelerating gap. Although the impedances of the power source and the feeding line are $50 \Omega$, the cavity impedance is designed as about $700 \Omega$ to moderate dissipated RF power. "Multifeed coupling" [3-4] has been developed to realise impedance matching between the cavity and the feeding line. The cavity impedance is set to about $50 \Omega$ per one FINEMET core to match the impedance of individual feeding lines.

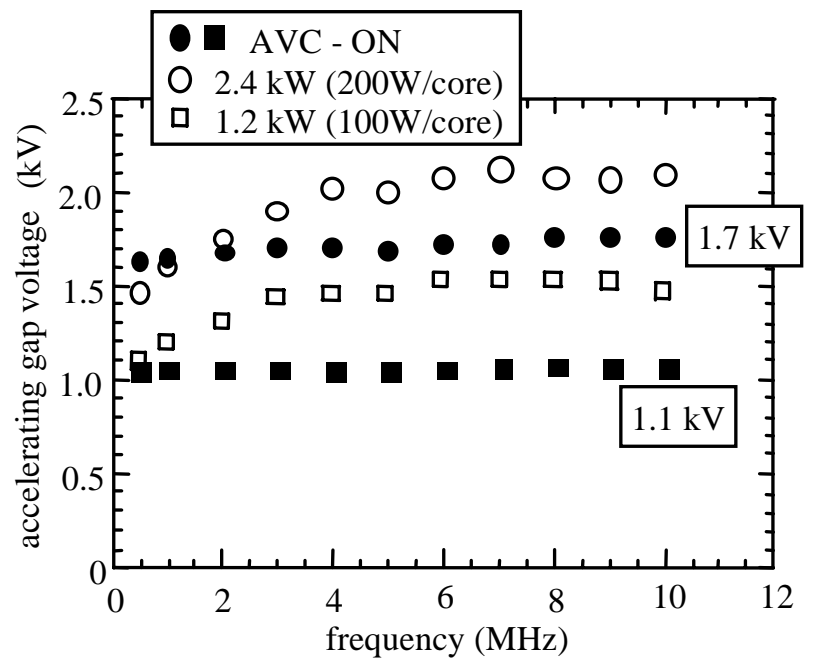

Figure 2: Results of high-power test of the RF cavity

The frequency dependence of the voltage standing wave ratio (VSWR) was measured with a network analyser. The VSWR was lower than 2 in the operation frequency range of $0.5-8.5 \mathrm{MHz}$, which meant power reflection from the cavity was less than $10 \%$. The results of a high-power test are shown in Fig. 2. An accelerating gap voltage higher than $1 \mathrm{kV}$ was attained with a supplied RF power of $1.2 \mathrm{~kW}$. The temperature rise of the FINEMET cores was $25{ }^{\circ} \mathrm{C}$ from room temperature of $20^{\circ} \mathrm{C}$, in the case of a supplied RF power of $2.4 \mathrm{~kW}$ (200W/core) which gave a gap voltage higher than $1.5 \mathrm{kV}$. The effectiveness of air cooling with the two blowers was confirmed. The gap voltage was limited only by the available RF power. Based on the experimental results of the testing cavity [12], operation should be possible at the supplied RF power of $800 \mathrm{~W} /$ core using only forced-air cooling. Then, the gap voltage higher than $3 \mathrm{kV}$ can be obtained.

\section{RF CONTROL SYSTEM}

We have developed an RF control system employing a direct digital synthesiser (DDS) as the reference signal generator for the cavity gap voltage, to realise stable and reproducible operation of the synchrotron. Figure 3 shows the block diagram. The DDS realises RF control system operations in a wide frequency range with low FM noise and highly stable and accurate centre frequency. Pattern data of the fundamental RF frequency are stored in the memory module and transferred to the DDS synchronously with the B-clock signal. The maximum clock-rate is $100 \mathrm{kHz}$, corresponding to a $0.2 \mathrm{G}$ increase and decrease of the bending magnetic field. The frequency converter generates the second harmonic signal and superposes it upon the fundamental one after amplitude and phase adjustment.

The RF control system employs the following four feedback loops: automatic voltage control loop (AVC loop), radial beam position control loop ( $\Delta \mathrm{R}$ loop), dipole and quadrupole synchrotron-oscillation suppression loops $(\Delta \Phi, 2 \mathrm{fs}$ loops). The AVC loop adjusts the cavity gap voltage to follow the designed value. The RF frequency is corrected with the $\Delta \mathrm{R}$ and $\Delta \Phi$ loops to reduce displacement of the beam orbit and to damp the dipole oscillation. The cavity gap voltage is slightly modulated with $2 \mathrm{fs}$ loop for the suppression of the quadrupole oscillation. The function of the voltage-controlled oscillator (VCO) is to generate an RF signal whose frequency is modulated by the $\Delta \mathrm{R}$ and $\Delta \Phi$ loops. The frequency converter inputs two RF signals generated by the DDS and the VCO, and outputs an RF signal with accurate acceleration frequency at the fundamental and the second harmonics. When the $\Delta \mathrm{R}$ and $\Delta \Phi$ loops are switched off, the oscillation frequency of the VCO is locked or switched to that of a crystal oscillator.

Before the beam acceleration test, we had checked the performance of the feedback loops with a signal generator to simulate the beam motions. Results of the performance tests have already been reported [5] and are summarised as follows: (1) The AVC loop realised voltage regulation within $5 \%$, as shown in Fig. 2. (2) The $\Delta \mathrm{R}$ loop reduced the orbit displacement from $60 \mathrm{~mm}$ to lower than $1 \mathrm{~mm}$. (3) The $\Delta \Phi$ loop suppressed the dipole oscillation of 5.7 $\mathrm{kHz}$ completely. (4) The $2 \mathrm{fs}$ loop damped the quadrupole oscillation of $8 \mathrm{kHz}$ rapidly.

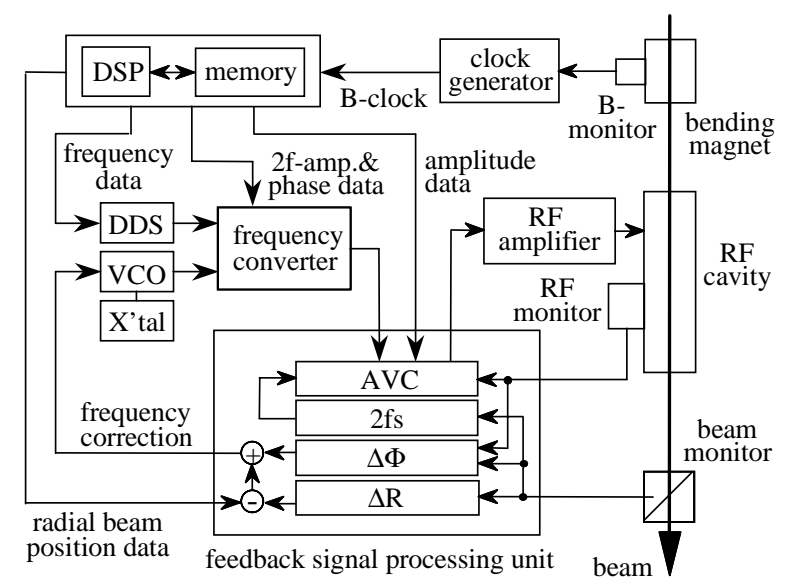

Figure 3: Block diagram of RF control system 


\section{BEAM ACCELERATION TEST}

We installed the RF accelerating system for a multipurpose synchrotron at the Wakasa-wan Energy Research Centre in Japan, and successfully accelerated three kinds of ion beams $\left(\mathrm{H}^{+}, \mathrm{He}^{2+}\right.$ and $\left.\mathrm{C}^{6+}\right)$. In the following, results of only the proton beam acceleration test are described. The injection energy was set at $10 \mathrm{MeV}$, which was determined by the terminal voltage of the tandem-type electrostatic accelerator. On the other hand, the flattop (extraction) energy was set at $200 \mathrm{MeV}$.

Effectiveness of the feedback system was confirmed at the beginning of commissioning. Synchrotron oscillations were observed at the dipole mode of $1.5-2.5 \mathrm{kHz}$ and at the quadrupole mode of 3-5 kHz. Those were suppressed with the $\Delta \Phi$ and 2 fs loops, and the beam intensity could be increased by $20-30 \%$. The radial beam position could be controlled with the $\Delta \mathrm{R}$ loop, as shown in Fig. 4, in which it was kept constant through the acceleration process. We updated the initial pattern data of the RF frequency by the $\Delta \mathrm{R}$ feedback correction.

After optimisation of the pattern data of the RF frequency and the gap voltage, the feedback system became unimportant, except for the AVC loop. The optimal gap voltage was around $200 \mathrm{~V}$ at the end of an adiabatic capture process, whereas it was set to $700 \mathrm{~V}$ in the final stage of the acceleration process. The beam intensity seemed to be limited by the space-charge effect, because a gap voltage higher than $300 \mathrm{~V}$ clearly deteriorated the capture efficiency. Then, we tried dualharmonics acceleration in the low energy region, namely, the capture process and the initial stage of the acceleration process. This successfully reduced the peak charge density of a bunched beam by $30 \%$ and enhanced the beam intensity at extraction by $40-50 \%$. Typical data are shown in Figs. 5-6; they were not been optimised in B-Q tracking. We finally attained the extraction beam intensity of $16 \mathrm{nC} /$ pulse by fine-tuning the working point of the synchrotron [6].

\section{FUTURE PLAN}

We began to design the present RF-control system five years ago. Since then, the technology for digital signal processing has made remarkable progress. Nowadays, digital feedback within a total latency time of $2 \mu$ s can be easily achieved in an inexpensive real-time control system. We plan to replace the original obsolete system by such a digital feedback system. The design study has already been finished for a multi-harmonic acceleration system. Triple-harmonics acceleration will enhance the beam intensity by a further $20 \%$. The bandwidth of the RF cavity is broad enough to accept the third-harmonic voltage.

\section{REFERENCES}

[1] K. Saito, J. Hirota and F. Noda, "An Untuned RF Cavity Loaded with Fe-based Nanocrystalline FINEMET Cores," Beam Science and Technology, Kyoto Univ. 2 (1997) 15-19.
[2] K. Saito et al., "FINEMET-core Loaded Untuned RF Cavity," Nucl. Instr. and Meth. A 402 (1998) 1-13.

[3] Y. Iwashita, "Ferro-magnetic Material Loaded Untuned RF Cavity for Synchrotron,” Jpn. J. Appl. Phys. 36 (1997) L727-L728.

[4] K. Saito et al., "An Untuned RF Cavity Using Multifeed Coupling," Nucl. Instr. and Meth. A 401 (1997) 133-143.

[5] K. Saito et al., "RF Acceleration System for Medical Synchrotron," Proc. 11th Symp. on Accelerator Science and Technology, Hyogo, Japan, 1997, pp. 197-199.

[6] K. Matsuda et al., "Beam Commissioning of a Multipurpose Compact Ion Synchrotron," in this conference.

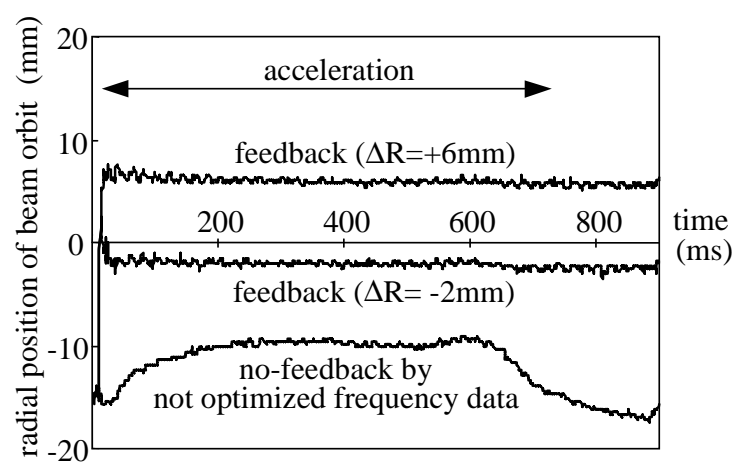

Figure 4: Radial beam position control by $\Delta \mathrm{R}$ feedback

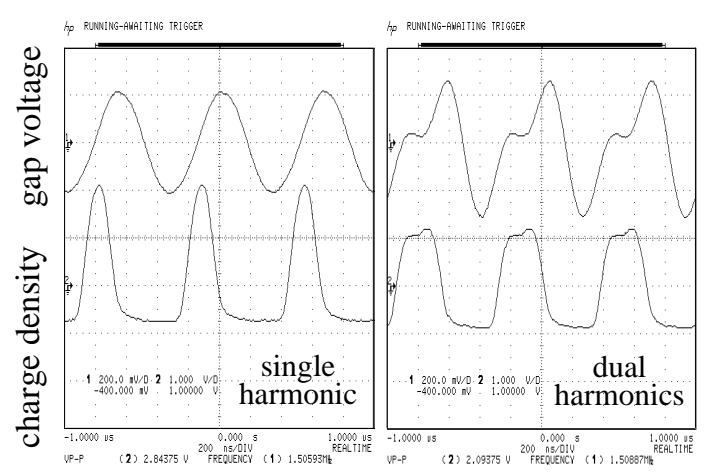

Figure 5: Peak density of bunched beam reduced by dual-harmonics acceleration

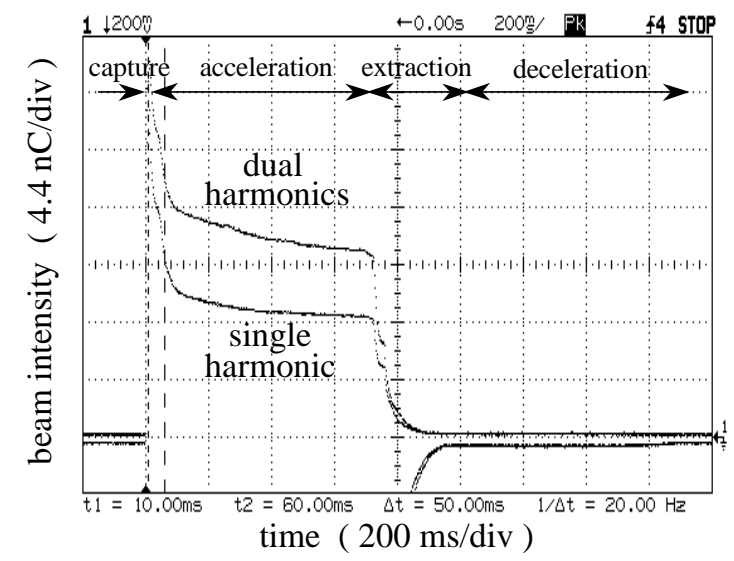

Figure 6: Beam intensity enhanced by dual-harmonics acceleration 